Research Article

\title{
Fractal Acoustic Metamaterials with Subwavelength and Broadband Sound Insulation
}

\author{
Yu Liu $\mathbb{D}^{1,2}$ Meng Chen $\mathbb{D}^{1,2}$ Wenshuai Xu, ${ }^{1,2}$ Tao Yang, ${ }^{1,2}$ Dongliang Pei, \\ Heng Jiang $\mathbb{D},{ }^{1,2}$ and Yuren Wang $\mathbb{D}^{1,2}$ \\ ${ }^{1}$ Key Laboratory of Microgravity, Institute of Mechanics, Chinese Academy of Sciences, Beijing 100190, China \\ ${ }^{2}$ University of Chinese Academy of Sciences, Beijing 100049, China \\ Correspondence should be addressed to Meng Chen; chenmeng@imech.ac.cn and Heng Jiang; hengjiang@imech.ac.cn
}

Received 15 March 2019; Revised 13 May 2019; Accepted 23 May 2019; Published 12 June 2019

Academic Editor: Chengzhi Shi

Copyright (C) 2019 Yu Liu et al. This is an open access article distributed under the Creative Commons Attribution License, which permits unrestricted use, distribution, and reproduction in any medium, provided the original work is properly cited.

\begin{abstract}
We construct new fractal acoustic metamaterials by coiling up space, which can allow subwavelength-scale and broadband sound insulation to be achieved. Using the finite element method and the S-parameter retrieval method, the band structures, the effective parameters, and the transmission losses of these acoustic metamaterials with different fractal orders are researched individually. The results illustrate that it is easy to form low-frequency bandgaps using these materials and thus achieve subwavelength-scale sound control. As the number of fractal orders increase, more bandgaps appear. In particular, in the $\Gamma X$ direction of the acoustic metamaterial lattice, more of these wide bandgaps appear in different frequency ranges, thus providing broadband sound insulation and showing promise for use in engineering applications.
\end{abstract}

\section{Introduction}

Low-frequency noise can cause a great deal of trouble in daily life because of the limitations of the mass law, which means that achieving subwavelength and broadband sound insulation is challenging. This has changed with the appearance of acoustic metamaterials [1-14], which can break the mass law and include materials such as locally resonant metamaterials [1-10], Helmholtz resonant metamaterials [11], and membrane metamaterials [12-14]. These metamaterials provide a way to control low-frequency sound waves with small-scale structures, but most of the above metamaterials only can achieve improved sound insulation within a single resonant frequency range, which thus provides only narrowband insulation. Researchers have adopted methods based on introduction of multiple coupled resonance units to broaden the insulation bandwidth [15-23]; however, most of these methods mainly broadened only their initial bandgaps. If a structure with multiple resonance modes can be developed to form multiple bandgaps, it will provide a promising basis for achievement of broadband sound insulation.
Through studies of labyrinthine fractal structures, researchers have found that multiple resonances appear in these structures [24-29] that can produce multiple bandgaps and thus broadband sound insulation. In these labyrinthine fractal structures, the sound waves propagate along the labyrinthine channels rather than in a straight line, which thus greatly increases their transmission paths and produces an ultraslow transmission effect. Because of the increased lengths of their transmission paths, these metamaterials have high refractive indexes and demonstrate extraordinary physical properties, including multiple bandgaps [24-29], negative refraction [30,31], and acoustic focusing [32, 33]. Liang et al. constructed an extreme acoustic metamaterial by coiling up the space using zigzag channels, which caused their material to have negative refraction and sound tunneling properties [30]. Cheng et al. proposed an ultrasparse metasurface for high reflection of low-frequency sound based on Mie resonance units [27]. Recently, Man et al. reported labyrinthine acoustic metamaterials with Hilbert fractal structures that could easily form low-frequency bandgaps and showed multibandgap properties [25]. When compared with the zigzag channels, the higher Hilbert 
fractal structures have longer transmission paths and thus have a higher refractive index that allows them to produce more excellent features. These results demonstrate that the properties of acoustic metamaterials will be improved by increasing the lengths of their transmission paths. Therefore, the design of fractal structures with longer paths is a promising and significant approach that may be helpful in controlling subwavelength-scale waves and achieving better broadband sound insulation. However, because of the physical limitation imposed by the tunnel width and thickness of the microscopic structure, it cannot be divided indefinitely, the fabricated fractal metamaterials consist of the geometry of only a certain scale, and the liquid channel of Hilbert fractal structures is not unlimited. It is meaningful to carry out a new design of the self-similar fractal acoustic metamaterials, which have longer effective transmission paths and show better acoustic properties.

In this paper, inspired by the Hilbert fractal acoustic metamaterials noted above, to achieve a longer effective length and get a better acoustic properties, we carried out a new design of self-similar fractal metamaterials by adopting the curve channels instead of the straight-line liquid channel adopted by existing fractal structures and determined the structural style of the self-similar fractal structure with curve channels. Through the new design, the fractal acoustic metamaterials with a self-similar fractal structure have more complex transmission paths and higher refractive indexes and are thus suitable for subwavelength-scale wave control and broadband sound insulation. By keeping the width unchanged, we emphatically study the influence of effective length to the acoustic properties of fractal metamaterials. Using the finite element method and the S-parameter retrieval method, the band structures, the effective properties, the transmission losses, and the sound pressure fields of the proposed acoustic metamaterials with their different fractal orders are calculated separately. Furthermore, the transmission losses in the $\Gamma X$ direction of the acoustic metamaterial lattice are studied in detail and show that the lattice contains greater numbers of wide bandgaps in different frequency ranges, thus causing broadband sound insulation and showing promise for use in engineering applications.

\section{Design of Fractal Acoustic Metamaterials}

Inspired by the Hilbert fractal acoustic metamaterials, in the text, to achieve a longer effective length and get better acoustic properties, we carried out a new design of self-similar fractal metamaterials, and the two-dimensional (2D) unit cells of the first-order, second-order, and third-order fractal structures are illustrated in Figures 1(a)-1(c), respectively. In our design, we adopt curves rather than the straight lines that are applied in traditional Hilbert fractal acoustic metamaterials, thus effectively increasing the total acoustic transmission length $\left(L_{\text {eff }}\right)$, particularly in the higher fractal units. As shown in Figure $1, R$ is the radius of the curves, the basic lattice constant of the fractal structure is $a(a=56 \mathrm{~mm})$, the transmission tunnel width is $d(d=3 \mathrm{~mm})$, and the relationships among the three parameters of the proposed first-order to third-order acoustic metamaterials can be expressed as follows:

$$
L_{\text {eff }}=2^{n}\left[2^{n-1}\left(\pi R_{n}+d\right)\right]+a,
$$

where

$$
R_{n}=\frac{a}{2^{n}}-\frac{d}{2}
$$

To get a higher fractal order, the tunnel width should be small enough, in our text, $d \ll a$; thus, the effective length can be simplified as $2^{n-1} \pi a$. This result firstly shows when the tunnel width is much smaller than the lattice constant, we can ignore the influence of tunnel width to the total effective length; thus we keep it unchanged in this text and emphatically study the influence of effective length to the acoustic properties of fractal metamaterials. Secondly, when acoustic waves propagate along the labyrinthine tunnels shown in the black arrows rather than in a straight line, we can effectively extend the effective wave propagation length $L_{\text {eff }}$ to be several times longer than a straight path within the same space; i.e., the acoustic wave propagates along the labyrinthine tunnels at an ultraslow equivalent speed when compared with the background medium. Therefore, the acoustic metamaterial can be understood to be an artificial medium with an ultraslow speed of sound and high relative refractive index $n_{\mathrm{r}}$. Furthermore, the wave propagation length $L_{\text {eff }}$ of the fractal metamaterials presented in this work is approximately $2^{n-1} \pi a$, which is $\pi / 2$ times longer than that of the previously reported Hilbert fractal acoustic metamaterials, which had an effective length $L_{\text {eff }}$ of $2^{n} a$, and through the new design, we can effectively extend the effective length of self-similar fractal metamaterials. It is thus helpful to design these labyrinthine acoustic metamaterials with ultraslow sound speeds and higher refractive indexes to achieve subwavelength-scale and broadband sound insulation.

\section{Band Structures of the Fractal Acoustic Metamaterials}

Acoustic waves are transmitted along the labyrinthine tunnels in the proposed metamaterials, and the harmonic wave equation in these tunnels can be expressed as

$$
\nabla \cdot\left(-\frac{1}{\rho_{0}} \nabla p\right)-\frac{w^{2} p}{\rho_{0} c_{0}}=0 .
$$

Here, $p$ is the air pressure, $w$ is the angular frequency, $\rho_{0}$ is the density of the air, and $c_{0}$ is the speed of sound in the air. We also set sound-hard boundary conditions at the air-solid interfaces and apply the Floquet-Bloch periodic conditions at the boundaries of the unit cells:

$$
\begin{gathered}
\frac{\partial p}{\partial n}=0, \\
p(x+a)=p(x) e^{-k a} .
\end{gathered}
$$

Here, $n$ is the normal vector of the air-solid interface and $k$ is the wave vector. In this work, the bandgaps were calculated using the finite element method via COMSOL 


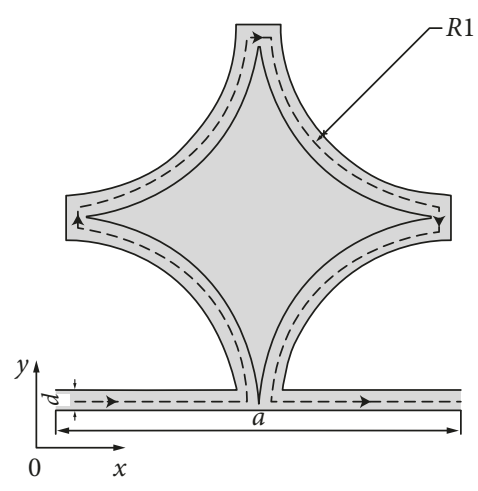

(a)

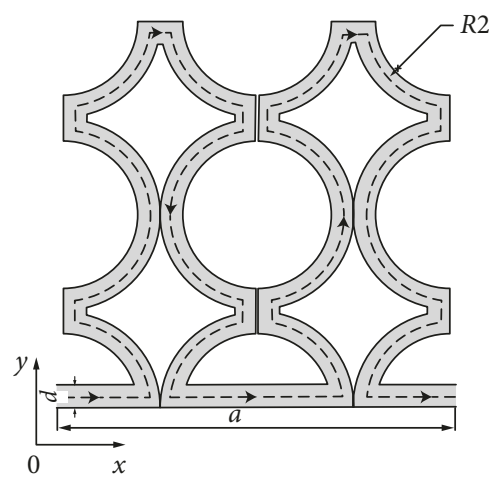

(b)

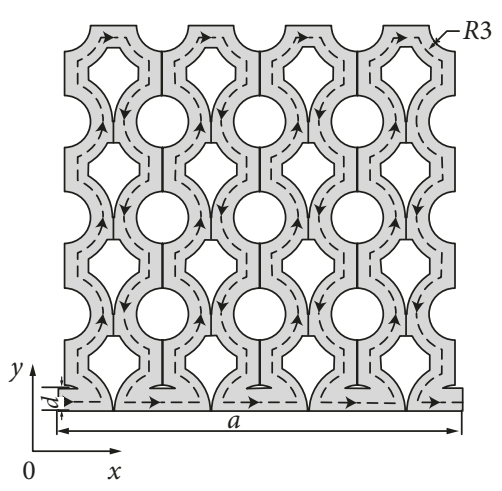

(c)

FIgURE 1: (a-c) Unit cells of the first-order to third-order fractal acoustic metamaterials.

Multiphysics software. By solving for the eigenfrequencies of the designed structures and the $w$ - $k$ dispersion relationships, we obtain the band structures of the fractal acoustic metamaterials, which are shown in Figure 2.

Figures $2(a)-2(c)$ show the band structures of the fractal acoustic metamaterials, ranging from the first order to the third order, where the horizontal axis represents the values of the wave vector $k$ along the borders $(\Gamma-X-M)$ of the triangular irreducible Brillouin zone of a square periodic lattice [20] and the vertical axis shows the frequency. In Figure 2(a), there are two total bandgaps $[840 \mathrm{~Hz}, 1110 \mathrm{~Hz}$ ] and $[2060 \mathrm{~Hz}$, $2106.4 \mathrm{~Hz}]$, and the proportion of these bandgaps is approximately $11.1 \%$ within the frequency range $[0 \mathrm{~Hz}, 2850 \mathrm{~Hz}]$. In Figure 2(b), there are four total bandgaps $[532.5 \mathrm{~Hz}$, $776.5 \mathrm{~Hz}],[980 \mathrm{~Hz}, 1067 \mathrm{~Hz}],[1926.2 \mathrm{~Hz}, 2148.3 \mathrm{~Hz}]$, and $[2750 \mathrm{~Hz}, 2845 \mathrm{~Hz}]$, and the proportion of these bandgaps is approximately $22.7 \%$ within the frequency range $[0 \mathrm{~Hz}$, $2850 \mathrm{~Hz}]$. In Figure 2(c), there are six total bandgaps [ $340 \mathrm{~Hz}$, $547 \mathrm{~Hz}],[646 \mathrm{~Hz}, 686 \mathrm{~Hz}],[1027 \mathrm{~Hz}, 1126 \mathrm{~Hz}],[1320 \mathrm{~Hz}$, $1411 \mathrm{~Hz}],[1912.5 \mathrm{~Hz}, 2055 \mathrm{~Hz}]$, and $[2518 \mathrm{~Hz}, 2750 \mathrm{~Hz}]$, and the proportion of these bandgaps is approximately $28.5 \%$ within the frequency range $[0 \mathrm{~Hz}, 2850 \mathrm{~Hz}]$.

From these results, we first find that, with the increasing fractal order, more total bandgaps occur and the proportion of these bandgaps also increases, particularly in the third order, where the proportion reaches $28.5 \%$ much higher than 20.53\% reported before; this indicates that the structure has better sound insulation properties. Second, the frequency ranges of the first bandgaps gradually become lower, particularly in the third order, where the range is $[340 \mathrm{~Hz}, 547 \mathrm{~Hz}]$ and the normalized frequency $\left(w a / c_{0}\right)$ range is $[0.056,0.09]$, which is lower than $[0.0762,0.1168]$ reported before and also much less than 1 , thus indicating that the designed fractal acoustic metamaterials can achieve subwavelength sound control. Furthermore, when compared with the zigzag channels and Hilbert channels, the transmission paths of the designed channels are longer and more complex, which is helpful in producing more bandgaps and can also help to reduce the size of the designed structure, which is significant in engineering applications.

\section{Calculation of Effective Parameters}

To aid in further understanding of the bandgaps formation diagram, we calculated the effective parameters of the designed fractal acoustic metamaterials using the S-parameter retrieval method [34]. The effective mass density and the bulk modulus relative to the air are calculated as follows:

$$
\begin{aligned}
& \rho_{\text {eff }}=\varepsilon \times n, \\
& B_{\text {eff }}=\frac{\varepsilon}{n} .
\end{aligned}
$$

According to the S-parameter retrieval method, $\varepsilon$ and $n$ can be expressed as follows:

$$
\begin{aligned}
& \varepsilon=\frac{r}{1-2 R+R^{2}-T^{2}}, \\
& n=\frac{-i \log x+2 \pi m}{k d},
\end{aligned}
$$

where

$$
\begin{aligned}
r & =\mp \sqrt{\left(R^{2}-T^{2}-1\right)^{2}-4 T^{2}}, \\
X & =\frac{\left(1-R^{2}+T^{2}+r\right)}{2 T} .
\end{aligned}
$$

In the above formulation, $R$ is the reflection coefficient of the structure and $T$ is the transmission coefficient of the structure; these coefficients were calculated using the COMSOL Multiphysics software and are plotted in Figures 3(a)-3(c). In this way, the effective parameters of the fractal metamaterials can be calculated, and the results are shown in Figures 3(d)-3(f). The results show that, within the frequency ranges of the bandgaps, there is a negative effective parameter that causes the formation of the bandgaps. For example, in the first-order fractal metamaterials, the bulk modulus $\left(B_{\text {eff }}\right)$ is negative in the first bandgap $[840 \mathrm{~Hz}$, $1110 \mathrm{~Hz}]$, while the mass density $\left(\rho_{\text {eff }}\right)$ is negative in the second bandgap $[2060 \mathrm{~Hz}, 2106.4 \mathrm{~Hz}]$. Furthermore, in Figures 3(e) and 3(f), the negative effective parameters appear in multiple frequency ranges, thus proving that the fractal structure contains multiple bandgaps and showing 


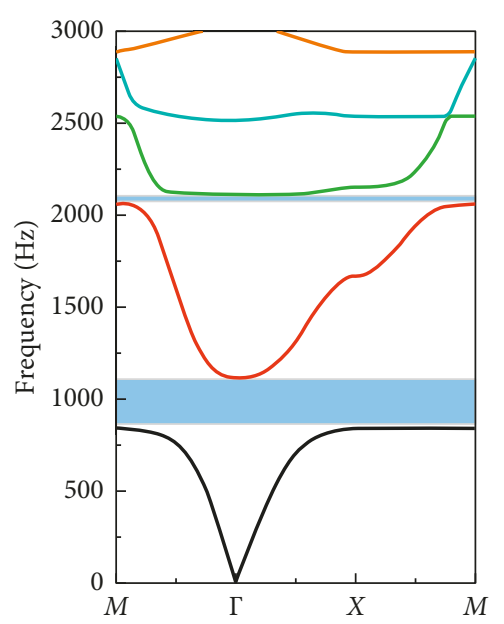

(a)

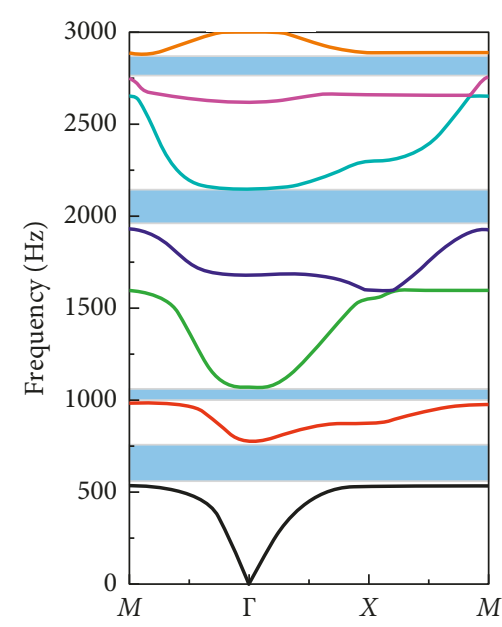

(b)

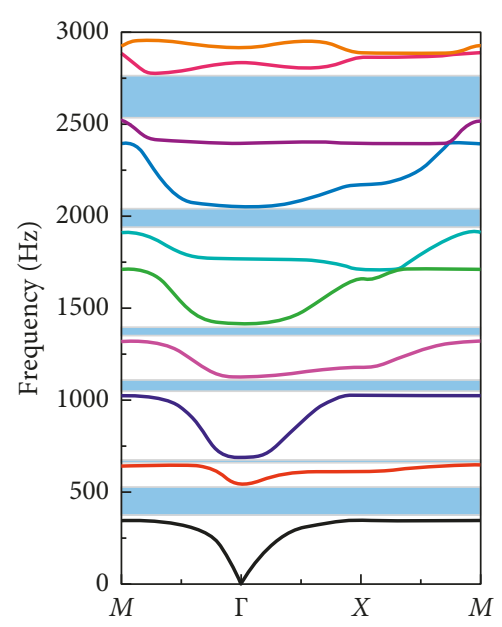

(c)

Figure 2: Band structures of fractal acoustic metamaterials. (a) Band structure of first-order fractal acoustic metamaterials in the frequency range $[0 \mathrm{~Hz}, 2850 \mathrm{~Hz}]$. (b) Band structure of second-order fractal acoustic metamaterials in the frequency range $[0 \mathrm{~Hz}, 2850 \mathrm{~Hz}]$. (c) Band structure of third-order fractal acoustic metamaterials in the frequency range $[0 \mathrm{~Hz}, 2850 \mathrm{~Hz}]$.

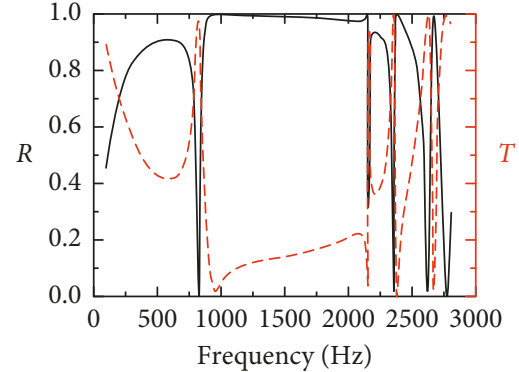

(a)
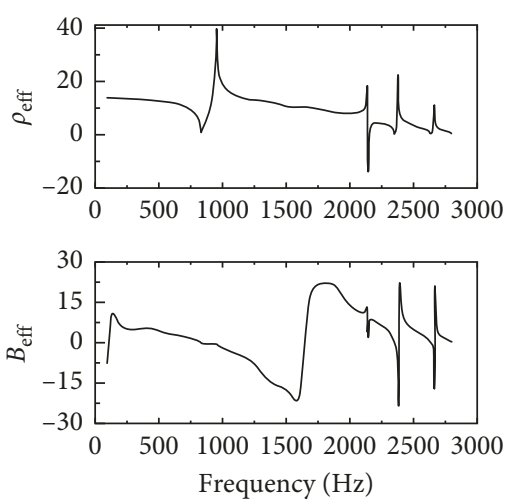

(d)

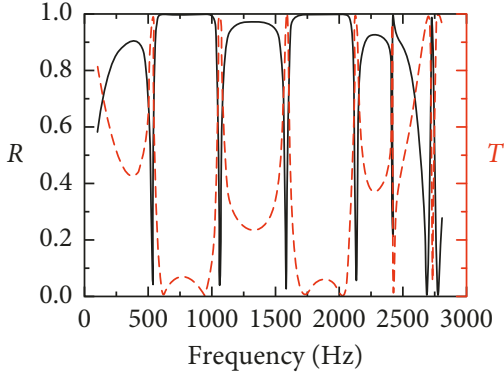

(b)
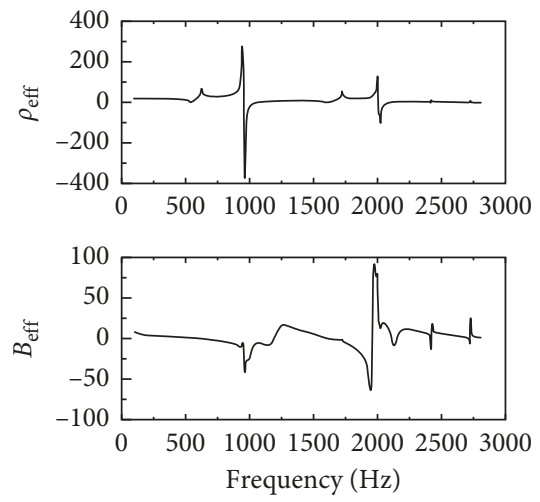

(e)

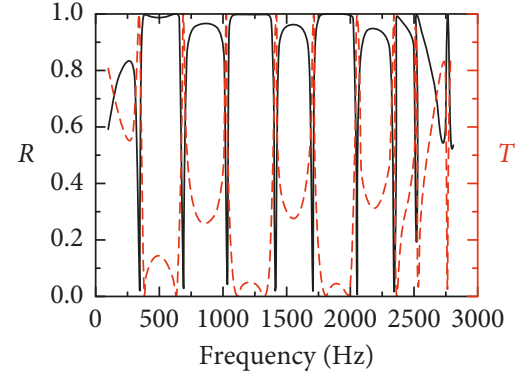

(c)
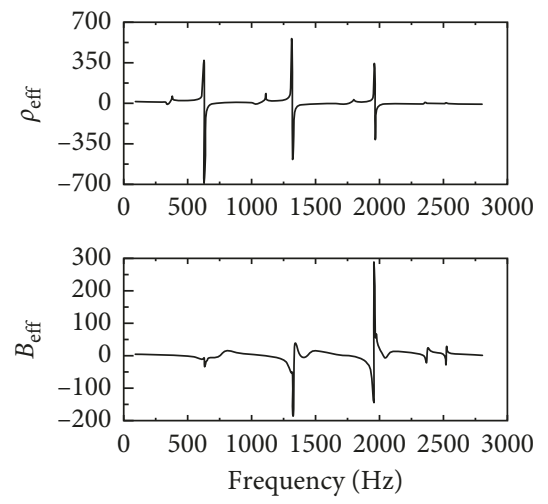

(f)

Figure 3: $(a-c)$ Reflection and transmission coefficients of the fractal acoustic structures from the first order to third order. (d-f) Effective parameter calculation results for the fractal acoustic structures from the first order to third order.

that it represents a promising way to achieve broadband sound insulation.

\section{Transmission Loss and Sound Insulation}

The calculation results for the band structures and the effective parameters of the designed materials show that there are multiple bandgaps in the fractal acoustic metamaterials that promise to allow broadband sound insulation to be achieved. To provide further verification of the broadband sound insulation performances of the designed materials, the transmission losses and band structures of the metamaterials in the $\Gamma X$ direction are calculated. Due to increase in the number of structure units, the properties of transmission losses will be better [24, 26, 29]; to get better sound insulation, we calculate the sound transmission losses of five units, with results as shown in Figures 4(a)-4(c). 


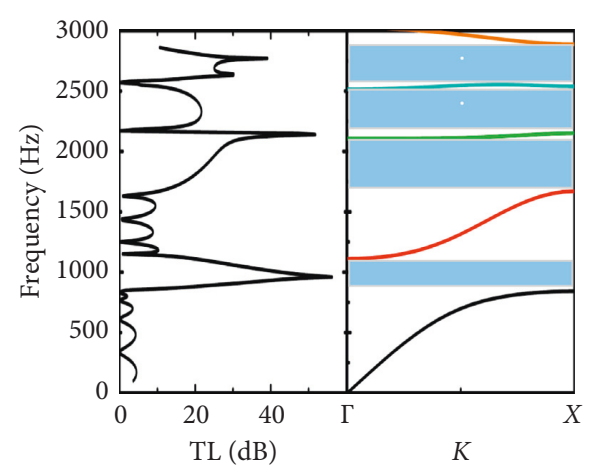

(a)

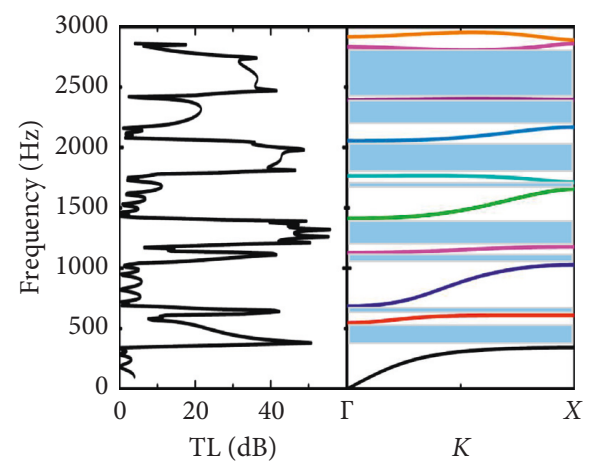

(c)

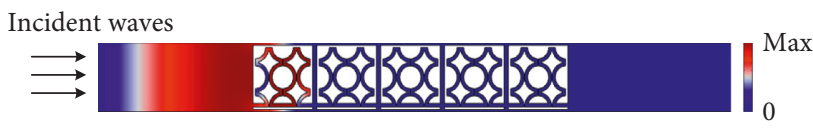

(e)

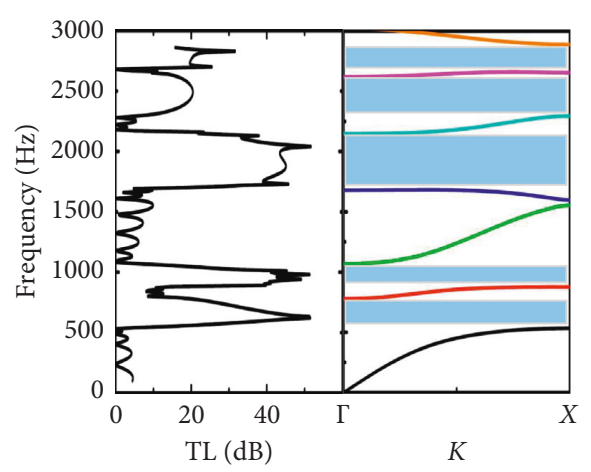

(b)

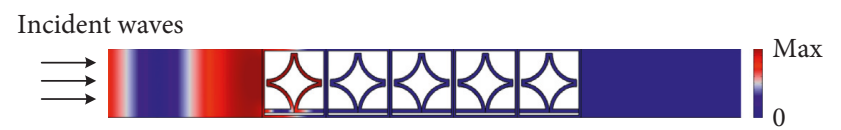

(d)

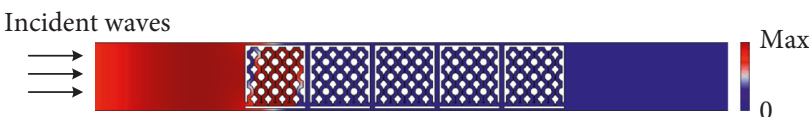

(f)

Figure 4: $(\mathrm{a}-\mathrm{c})$ Transmission losses of the fractal acoustic structures with five units, from the first order to third order. (d) Sound pressure field of the first order at $960 \mathrm{~Hz}$. (e) Sound pressure field of the second order at $620 \mathrm{~Hz}$. (f) Sound pressure field of the third order at $380 \mathrm{~Hz}$.

First, the calculation results show that there are more bandgaps in the $\Gamma X$ direction and that the higher transmission losses appear within the ranges of these bandgaps. The ranges of bandgaps and higher transmission losses agree well with each other. Second, with the increasing fractal order, the number of bandgaps increases and the proportions of the bandgaps within their frequency ranges also increase, from $47.8 \%$ to $52.9 \%$ to $61.1 \%$ in the examples shown. Furthermore, the lowest normalized frequencies of the second-order and third-order transmission losses are 0.1 and 0.063 , respectively, which are both much less than 1 ; this indicates that we can achieve subwavelength sound insulation using fractal acoustic metamaterials with structural sizes that are much smaller than the wavelength in the required frequency ranges.

We also calculated the sound pressure field distributions of the fractal acoustic metamaterials from the first order to the third order. The sound pressure field distributions at $960 \mathrm{~Hz}, 620 \mathrm{~Hz}$, and $380 \mathrm{~Hz}$ are plotted in Figures $4(\mathrm{~d})-4(\mathrm{f})$, respectively. The results show that the acoustic fractal metamaterials at the subwavelength sound scale can reduce the sound pressure fields by $56.2 \mathrm{~dB}$, $51.5 \mathrm{~dB}$, and $50.8 \mathrm{~dB}$, which is helpful for control of low- frequency acoustic signals when using the designed fractal acoustic metamaterials.

\section{Conclusions}

In this work, we have designed a new type of self-similar fractal acoustic metamaterial with space-coiling curls and have systematically studied the subwavelength-scale and broadband sound insulation properties of these materials by calculating their band structures, effective parameters, transmission losses, and sound pressure fields. First, the results show that as the fractal order increases, more bandgaps appear, particularly in the $\Gamma X$ direction; in addition, there are more directional bandgaps and high transmission loss peaks appear in these bandgaps, thus proving that the proposed structures can achieve broadband sound insulation. Second, with the increasing fractal order, the first bandgap becomes lower, particularly in the third order, where the normalized frequency in the $\Gamma X$ direction is 0.063 ; this is much smaller than 1 , thus proving that the designed structures can achieve subwavelength-scale sound control. Furthermore, the frequency ranges of the negative effective parameters are well matched with the bandgaps, which is the reason for the 
production of these bandgaps. These results show that compared with the self-similar fractal structure reported before, the new designed structure has a longer effective length and more extraordinary properties for broadband and low-frequency sound insulation, and they are promising for applications in engineering applications. In the future work, to make the new designed structure show the best acoustic properties, we will consider the influence of tunnel width and the structure will be further optimized.

\section{Data Availability}

The numerical data used to support the findings of this study are available from the corresponding author upon request.

\section{Conflicts of Interest}

The authors declare that there are no conflicts of interest regarding the publication of this article.

\section{Acknowledgments}

The authors acknowledge project supported by the National Natural Science Foundation of China (Grant no. 11602269), the Strategic Priority Research Program of the Chinese Academy of Sciences (Grant no. XDB22040301), and the Research Program of Beijing (Grant nos. Z161100002616034 and Z171100000817010).

\section{References}

[1] Z. Liu, X. Zhang, Y. Mao et al., "Locally resonant sonic materials," Science, vol. 289, no. 5485, pp. 1734-1736, 2000.

[2] Z. Liu, C. T. Chan, and P. Sheng, "Three-component elastic wave band-gap material," Physical Review B, vol. 65, no. 16, article 165116, 2002.

[3] M. Hirsekorn, "Small-size sonic crystals with strong attenuation bands in the audible frequency range," Applied Physics Letters, vol. 84, no. 17, pp. 3364-3366, 2004.

[4] G. Wang, X. Wen, J. Wen, L. Shao, and Y. Liu, "Twodimensional locally resonant phononic crystals with binary structures," Physical Review Letters, vol. 93, no. 15, article 154302, 2004.

[5] J.-C. Hsu and T.-T. Wu, "Lamb waves in binary locally resonant phononic plates with two-dimensional lattices," Applied Physics Letters, vol. 90, no. 20, article 201904, 2007.

[6] R. Sainidou, B. Djafari-Rouhani, Y. Pennec, and J. O. Vasseur, "Locally resonant phononic crystals made of hollow spheres or cylinders," Physical Review B, vol. 73, no. 2, article 024302, 2006.

[7] Y. Pennec, B. Djafari-Rouhani, H. Larabi, J. O. Vasseur, and A. C. Hladky-Hennion, "Low-frequency gaps in a phononic crystal constituted of cylindrical dots deposited on a thin homogeneous plate," Physical Review B, vol. 78, no. 10, article 104105, 2008.

[8] T.-T. Wu, Z.-G. Huang, T.-C. Tsai, and T.-C. Wu, "Evidence of complete band gap and resonances in a plate with periodic stubbed surface," Applied Physics Letters, vol. 93, no. 11, article 111902, 2008

[9] M. Oudich, M. B. Assouar, and Z. Hou, "Propagation of acoustic waves and waveguiding in a two-dimensional locally resonant phononic crystal plate," Applied Physics Letters, vol. 97, no. 19, article 193503, 2010.

[10] A. Khelif, Y. Achaoui, S. Benchabane, V. Laude, and B. Aoubiza, "Locally resonant surface acoustic wave band gaps in a two-dimensional phononic crystal of pillars on a surface," Physical Review B, vol. 81, no. 21, article 214303 1-7, 2010.

[11] N. Fang, D. Xi, J. Xu et al., "Ultrasonic metamaterials with negative modulus," Nature Materials, vol. 5, no. 6, pp. 452-456, 2006.

[12] Z. Yang, J. Mei, M. Yang, N. H. Chan, and P. Sheng, "Membrane-type acoustic metamaterial with negative dynamic mass," Physical Review Letters, vol. 101, no. 20, article 204301, 2008.

[13] J. Li, X. Zhou, G. Huang, and G. Hu, "Acoustic metamaterials capable of both sound insulation and energy harvesting," Smart Materials and Structures, vol. 25, no. 4, article 045013, 2016.

[14] W. Zhou, B. Wu, Muhammad et al., "Actively tunable transverse waves in soft membrane-type acoustic metamaterials," Journal of Applied Physics, vol. 123, no. 16, article 165304, 2018.

[15] S. Zhang and J. Cheng, "Existence of broad acoustic bandgaps in three-component composite," Physical Review B, vol. 68, no. 24, article 245101, 2003.

[16] H. Jiang, Y. Wang, M. Zhang et al., "Locally resonant phononic woodpile: a wide band anomalous underwater acoustic absorbing material," Applied Physics Letters, vol. 95, no. 10, article 104101, 2009.

[17] J. P. Xia, X. T. Zhang, H. X. Sun, S.-Q. Yuan, J. Qian, and Y. Ge, "Broadband tunable acoustic asymmetric focusing lens from dual-layer metasurfaces," Physical Review Applied, vol. 10, no. 1, article 014016, 2018.

[18] H. Long, Y. Cheng, and X. Liu, "Asymmetric absorber with multiband and broadband for low-frequency sound," Applied Physics Letters, vol. 111, no. 14, article 143502, 2017.

[19] Y. Tang, S. Ren, H. Meng et al., "Hybrid acoustic metamaterial as super absorber for broadband low-frequency sound," Scientific Reports, vol. 7, no. 1, article 43340, 2017.

[20] S.-H. Park, "Acoustic properties of micro-perforated panel absorbers backed by Helmholtz resonators for the improvement of low-frequency sound absorption," Journal of Sound and Vibration, vol. 332, no. 20, pp. 4895-4911, 2013.

[21] C. R. Liu, J. H. Wu, X. Chen, and F. Ma, "A thin low-frequency broadband metasurface with multi-order sound absorption," Journal of Physics D: Applied Physics, vol. 52, no. 10, article 105302, 2019

[22] Y. Liao, X. Zhou, Y. Chen, and G. Huang, "Adaptive metamaterials for broadband sound absorption at low frequencies," Smart Materials and Structures, vol. 28, no. 2, article 025005, 2018.

[23] J. Yang, J. S. Lee, and Y. Y. Kim, "Multiple slow waves in metaporous layers for broadband sound absorption," Journal of Physics D: Applied Physics, vol. 50, no. 1, article 015301, 2017.

[24] J. Liu, L. Li, B. Xia, and X. Man, "Fractal labyrinthine acoustic metamaterial in planar lattices," International Journal of Solids and Structures, vol. 132-133, pp. 20-30, 2018.

[25] X. Man, T. Liu, B. Xia, Z. Luo, L. Xie, and J. Liu, "Space-coiling fractal metamaterial with multi-bandgaps on subwavelength scale," Journal of Sound and Vibration, vol. 423, pp. 322-339, 2018.

[26] A. O. Krushynska, F. Bosia, M. Miniaci, and N. M. Pugno, "Spider web-structured labyrinthine acoustic metamaterials 
for low-frequency sound control," New Journal of Physics, vol. 19, no. 10, article 105001, 2017.

[27] Y. Cheng, C. Zhou, B. G. Yuan, D. J. Wu, Q. Wei, and X. J. Liu, "Ultra-sparse metasurface for high reflection of lowfrequency sound based on artificial Mie resonances," $\mathrm{Na}$ ture Materials, vol. 14, no. 10, pp. 1013-1019, 2015.

[28] A. O. Krushynska, F. Bosia, and N. M. Pugno, "Labyrinthine acoustic metamaterials with space-coiling channels for lowfrequency sound control," Acta Acustica United with Acustica, vol. 104, no. 2, pp. 200-210, 2018.

[29] M. Miniaci, A. Krushynska, A. S. Gliozzi, N. Kherraz, F. Bosia, and N. M. Pugno, "Design and fabrication of bioinspired hierarchical dissipative elastic metamaterials," Physical Review Applied, vol. 10, no. 2, article 024012, 2018.

[30] Z. Liang and J. Li, "Extreme acoustic metamaterial by coiling up space," Physical Review Letters, vol. 108, no. 11, article 114301, 2012.

[31] Z. Liang, T. Feng, S. Lok et al., "Space-coiling metamaterials with double negativity and conical dispersion," Scientific Reports, vol. 3, no. 1, p. 1614, 2013.

[32] Y. Li, B. Liang, X. Tao, X.-F. Zhu, X.-Y. Zou, and J.-C. Cheng, "Acoustic focusing by coiling up space," Applied Physics Letters, vol. 101, no. 23, article 233508, 2012.

[33] L. Zigoneanu, B. I. Popa, and S. A. Cummer, "Design and measurements of a broadband two-dimensional acoustic lens," Physical Review B, vol. 84, no. 2, article 024305, 2011.

[34] V. Fokin, M. Ambati, C. Sun, and X. Zhang, "Method for retrieving effective properties of locally resonant acoustic metamaterials," Physical Review B, vol. 76, no. 14, article 144302, 2007. 


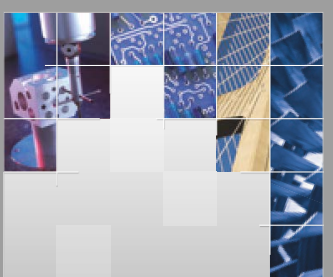

\section{Enfincering}
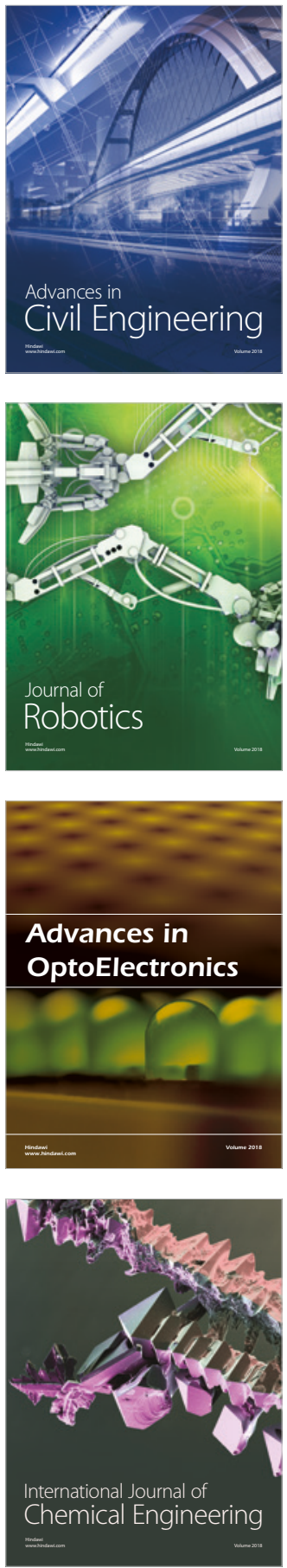

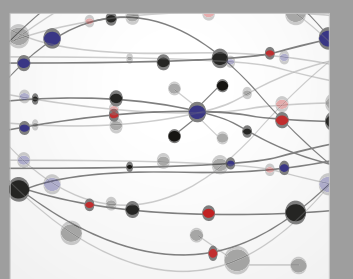

\section{Rotating \\ Machinery}

The Scientific World Journal

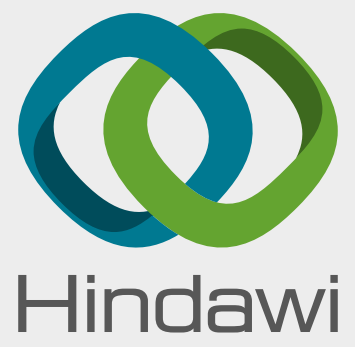

Submit your manuscripts at

www.hindawi.com
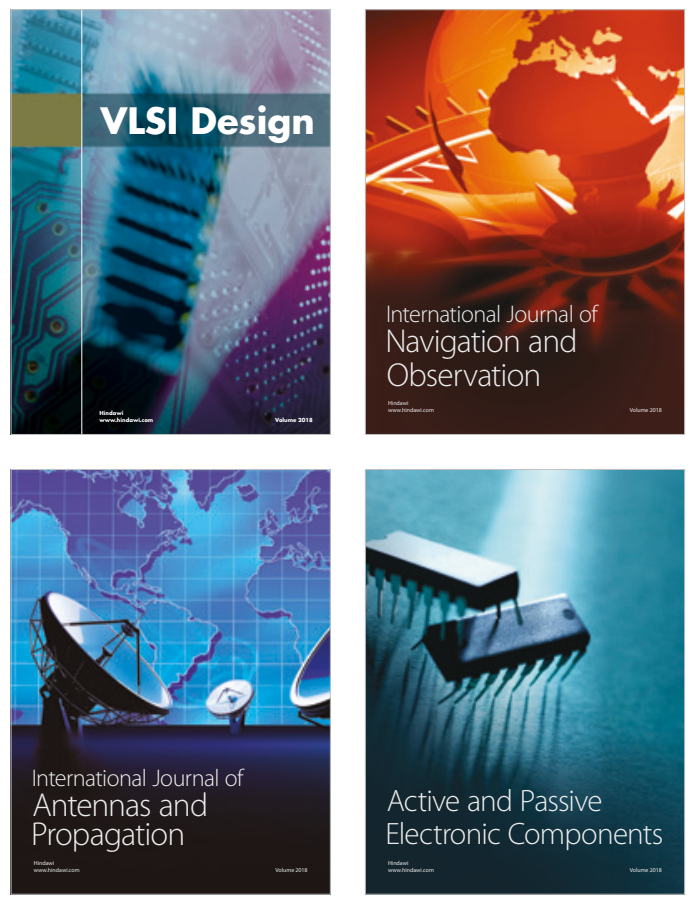
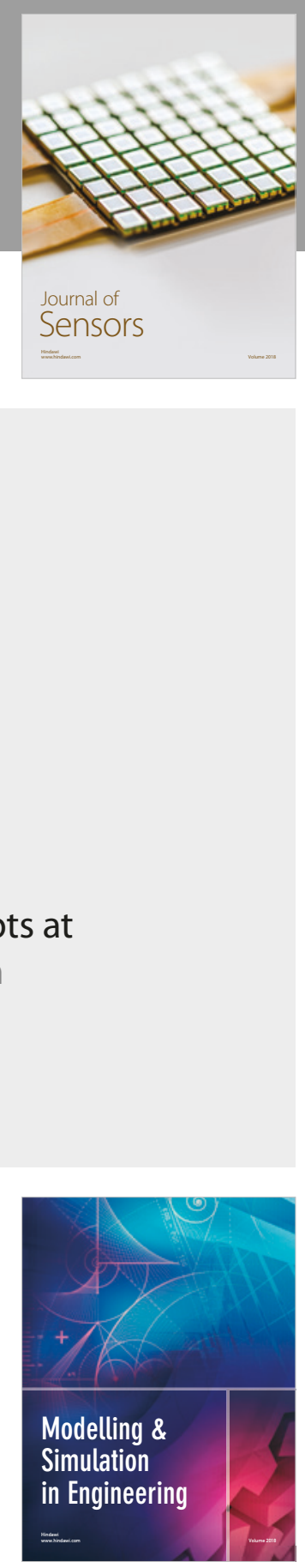

\section{Advances \\ Multimedia}
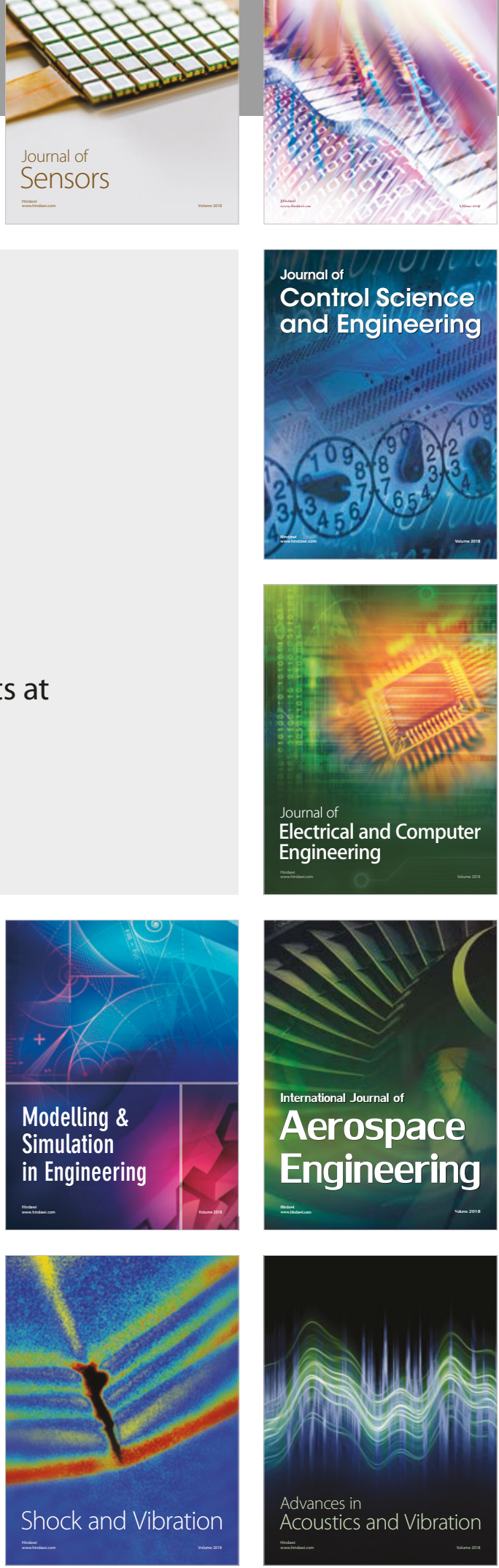